\title{
NEAR COHERENCE OF FILTERS. II: APPLICATIONS TO OPERATOR IDEALS, THE STONE-ČECH REMAINDER OF A HALF-LINE, ORDER IDEALS OF SEQUENCES, AND SLENDERNESS OF GROUPS
}

\author{
ANDREAS BLASS
}

\begin{abstract}
The set-theoretic principle of near coherence of filters (NCF) is known to be neither provable nor refutable from the usual axioms of set theory. We show that NCF is equivalent to the following statements, among others: (1) The ideal of compact operators on Hilbert space is not the sum of two smaller ideals. (2) The Stone-Čech remainder of a half-line has only one composant. (This was first proved by J. Mioduszewski.) (3) The partial ordering of slenderness classes of abelian groups, minus its top element, is directed upward (and in fact has a top element). Thus, all these statements are also consistent and independent.
\end{abstract}

1. Introduction. The primary purpose of this paper is to present applications, in rather diverse areas of mathematics, of the set-theoretic principle of near coherence of filters, NCF, that was introduced in [6] and proved to be consistent in [7]. The remainder of this introduction will give a brief survey of these applications. Terminology used without explanation here will be explained either in §2, which also contains a review of NCF and related information, or in the later sections, $\$ 3$ through $\S 6$, that deal with the individual applications. These later sections can be read independently of each other except that $\$ 6$ depends on $\$ 5$.

NCF first arose in my joint work [5] with G. Weiss on a problem of A. Brown, C. Pearcy, and N. Salinas [9]: Is the ideal of compact operators, in the ring of bounded operators on Hilbert space, the sum of two properly smaller ideals? We proved in [5] that an affirmative answer follows from the continuum hypothesis and in fact from any of several weaker hypotheses, the weakest of which, called $(* *)$ in [5, p. 414], is precisely the negation of NCF. The proof that this hypothesis suffices for the result of [5], and also the (more recent) proof of the converse, are given in $\$ 3$ below.

Wanting to prove $(* *)$ and thus remove all special hypotheses from [5], I mentioned the question to numerous set theorists. At the 1977 Toronto meeting in honor of Rothberger, E. van Douwen informed me that $(* *)$ was the hypothesis needed to prove, by the method of Rudin [19], that the indecomposable continuum $\beta \mathbf{R}^{+}-\mathbf{R}^{+}$, the Stone-Čech remainder of a half-line, has more than one composant. It turned out that this hypothesis is needed not just for Rudin's proof but for the

Received by the editors February 4, 1986.

1980 Mathematics Subject Classification (1985 Revision). Primary 03E05, 03C20, 03E35, 54D40, 03E75; Secondary 54F20, 47D25, 20K20. 
result itself. In other words, $\beta \mathbf{R}^{+}-\mathbf{R}^{+}$has only one composant if and only if NCF holds. I learned later (at Oberwolfach in 1985) that this equivalence had been obtained earlier by J. Mioduszewski [15] as a consequence of his general study [16] of $\beta \mathbf{R}$. Nevertheless, a proof is given in $\S 4$ below, which avoids this general theory and relies instead on the methods of Bellamy [2] and Rudin [19].

In May, 1984, I gave a series of lectures about NCF in Berlin. My audience included B. Wald, who conjectured that NCF might be related to his joint work with R. Göbel $[12,13]$ on the partial ordering $P$ of growth types (or, equivalently, of slenderness classes of abelian groups). They had shown that $\mathbf{P}$ has at least four elements. Theorem 6.3 below implies that, if $\mathbf{P}$ has only four elements, then NCF holds. The converse is still an open problem, but Theorem 6.3 does provide some equivalents for NCF in terms of $\mathbf{P}$. $\$ 6$ also includes some results about $\mathbf{P}$ that are not directly related to NCF. All the material in $\$ 6$ is based on a similar analysis in $\S 5$ of a somewhat larger ordering than $\mathbf{P}$, which seems combinatorially more natural and easier to handle, but which lacks the connection to group theory that motivated the study of $\mathbf{P}$.

2. Notation and preliminaries. We write $\omega$ for the set of natural numbers and $\omega \nearrow \omega$ for the set of nondecreasing functions from $\omega$ to $\omega$. A function $h: \omega \rightarrow \omega$ (not necessarily monotone) is said to be finite-to-one if $h^{-1}\{n\}$ is finite for all $n$.

LEMMA 2.1. If $h: \omega \rightarrow \omega$ is finite-to-one, then $\omega$ can be partitioned into finite intervals so that any $n$ and its image $h(n)$ are either in the same interval or in adjacent intervals of the partition.

Proof. Let the first interval in the partition be $\{0\}$. After an interval $[a, b]$ in the partition has been defined, let the next one be $[b+1, c]$ where $c$ is the largest element of $\{b+1\} \cup h([a, b]) \cup h^{-1}([a, b])$.

The sum of two functions $\omega \rightarrow \omega$ is defined, as usual, by $(f+g)(n)=f(n)+$ $g(n)$. Other operations, for example the binary maximum operation, are similarly defined for functions.

If $A$ is an infinite subset of $\omega$ and $n \in \omega$, then $\operatorname{next}(A, n)$ denotes the smallest $a \in A$ such that $a \geqslant n$.

By an ultrafilter, we always mean a nonprincipal ultrafilter on $\omega$. For background information on ultrafilters, see [10]. In addition to the definition, we shall need the following characterization of ultrafilters, whose proof is elementary.

LEMMA 2.2. A nonempty family of subsets of $\omega$ is an ultrafilter if and only if it is closed under supersets, it contains no two disjoint sets, it contains no finite sets, and, whenever it contains the union of two disjoint sets, then it contains one of those two sets.

Ultrafilters can be identified with points in the Stone-Čech remainder $\beta \omega-\omega$ of the discrete topological space $\omega$; ultrafilter $U$ is identified with the unique point that is in the closure, in $\beta \omega$, of every $X \in U$. 
An ultrafilter $U$ is a $Q$-point if every finite-to-one function $h: \omega \rightarrow \omega$ is one-to-one on some set in $U$. For this it suffices that every such $h$ be at-most-two-to-one on some $X \in U$, for then $X=X_{1} \cup X_{2}$ with $h$ one-to-one on each $X_{i}$, and one of the $X_{i}$ is in $U$. (At-most- $n$-to-one, for any fixed $n$, also suffices, but we shall not need this.)

LemmA 2.3. If $U$ is a Q-point and $f: \omega \rightarrow \omega$, then there is $X \in U$ such that, whenever $x<y$ are in $X$, then $f(x)<y$.

Proof. Replacing $f(x)$ with $\max \{f(x), x\}$, we may assume that $f$ is finite-to-one and apply Lemma 2.1 to it. Applying the definition of $Q$-point with an $h$ that is constant on exactly the intervals of the partition given by Lemma 2.1, we obtain an $X_{0} \in U$ that contains at most one point from each of these intervals. Partition $X_{0}$ into $X_{1}$ and $X_{2}$ by putting every second member of $X_{0}$ into $X_{1}$. Then no two elements of $X_{1}$ are in the same interval or adjacent intervals of our partition, and the same holds of $X_{2}$. As $U$ is an ultrafilter and contains $X_{0}$, it contains either $X_{1}$ or $X_{2}$, and this set clearly serves as the desired $X$.

If $U$ is an ultrafilter and $f: \omega \rightarrow \omega$ is not constant on any set in $U$, then $f(U)$ is the ultrafilter

$$
f(U)=\left\{X \subseteq \omega \mid f^{-1}(X) \in U\right\} .
$$

$f(U)$ contains all the sets $f(Y)$ for $Y \in U$, and it is generated by these sets, i.e., every $X \in f(U)$ has a subset of the form $f(Y)$ with $Y \in U$. If $f$ and $g$ agree when restricted to some set in $U$, then $f(U)=g(U)$. If $f$ is one-to-one on some set in $U$, then there exists a bijection $g: \omega \rightarrow \omega$ that agrees with $f$ on a (possibly smaller) set in $U$; we say that $f(U)=g(U)$ is isomorphic to $U$. In particular, if $U$ is a $Q$-point and $f$ is finite-to-one, then $f(U)$ is isomorphic to $U$ and is therefore also a $Q$-point.

Two ultrafilters, $U$ and $V$, are said to be cofinally equivalent if there are finite-to-one functions $f$ and $g$ with $f(U)=g(V)$. It is shown in [6], where this concept was introduced, that one can equivalently require in the definition that $f=g$ and that $f$ is nondecreasing.

LEMMA 2.4. If $U$ and $V$ are cofinally equivalent and if $V$ is a $Q$-point, then $V=h(U)$ for some finite-to-one $h: \omega \rightarrow \omega$.

Proof. By hypothesis, we have $f(U)=g(V)$ with $f$ and $g$ finite-to-one. As $V$ is a $Q$-point, $g$ is one-to-one on a set in $V$ and is therefore equal, on some set in $V$, to a bijection $b: \omega \rightarrow \omega$. Then $V=b^{-1} g(V)=b^{-1} f(U)$, so $h=b^{-1} f$ works.

LEMMA 2.5. Let $U$ and $V$ be ultrafilters that are not cofinally equivalent, and let $\omega$ be partitioned into finite intervals. Then there exist $X \in U$ and $Y \in V$ such that no union of two adjacent intervals from the partition intersects both $X$ and $Y$.

Proof. Let $h \in \omega \nearrow \omega$ be the function that is constant with value $n$ on the $n$th interval of our partition, for each $n$. Let $h^{-}(x)$ (resp. $h^{+}(x)$ ) be the result of rounding $h(x) / 2$ down (resp. up) to an integer. $h^{-}$is finite-to-one, so, as $U$ and $V$ are not cofinally equivalent, $h^{-}(U) \neq h^{-}(V)$. So there are $X^{-} \in U$ and $Y^{-} \in V$ with 
$h^{-}\left(X^{-}\right)$disjoint from $h^{-}\left(Y^{-}\right)$. Similarly, find $X^{+} \in U$ and $Y^{+} \in V$ with disjoint $h^{+}$-images. Then $X=X^{+} \cap X^{-}$and $Y=Y^{+} \cap Y^{-}$are as desired, because, on every union of two adjacent intervals, either $h^{-}$or $h^{+}$is constant.

Near coherence of filters, abbreviated NCF, is the assertion that every two ultrafilters are cofinally equivalent. Its name comes from the following equivalent formulation given in [6, Theorem 8].

LEMMA 2.6. NCF holds if and only if, for every two filters $F_{1}, F_{2}$ on $\omega$ that contain all cofinite sets, there exists a partition of $\omega$ into finite intervals such that, for each $X_{1} \in F_{1}$ and $X_{2} \in F_{2}$, some interval of the partition meets both $X_{1}$ and $X_{2}$.

In $\$ \S 5$ and 6 , we shall need some facts about non-standard models of arithmetic and their relationship with ultrafilters. General references for this material are $[\mathbf{1 , ~ 8 ,}$ 17, 18]. By a model of arithmetic, we mean an elementary extension $M$ of the standard model consisting of $\omega$ and all (finitary) relations and functions on it. That is, $M$ is a superset of $\omega$, equipped with a canonical extension to $M$ of every relation and function on $\omega$, such that all first-order sentences true in $\omega$ remain true in $M$. We usually use the same symbol for a relation or function on $\omega$ and its extension to $M$. These extensions are called the standard relations and functions on $M$. In the case of unary relations $A$, we use the notation $x \in A$ synonymously with $A(x)$.

Any element $a$ in a model $M$ of arithmetic generates a submodel consisting of all the elements $f(a)$ where $f$ ranges over (the canonical extensions to $M$ of) all unary functions $f: \omega \rightarrow \omega$. This submodel is itself a model of arithmetic and is canonically isomorphic to the ultrapower $U$-prod $\omega$, where $U$ is the ultrafilter

$$
U=\{X \subseteq \omega \mid a \in X\},
$$

called the (complete) type of $a$ (in $M$ ), and where the isomorphism sends an equivalence class $[f] \in U$-prod $\omega$ to $f(a) \in M$. (Note that, in the definition of $U$, the first " $X$ " refers to a subset of $\omega$, the second to its extension in $M$.)

Two elements of $M$ that generate the same submodel are said to be in the same constellation. This equivalence relation can also be defined by saying that one element is sent to the other by a standard bijection or by saying that each of the two elements is the image of the other by a standard function. The weaker equivalence relation saying only that each of two elements is majorized (with respect to the canonical extension of the standard order of $\omega$ ) by a standard function of the other, i.e. that the submodels generated by the two elements are cofinal in the same initial segment of $M$, is expressed by saying that the two elements are in the same sky. Each sky is a union of constellations and is an order-convex set in $M$; thus, the set of skies inherits from $M$ a natural ordering. The union of any sky with all the skies below it is a submodel of $M$; thus, each sky is the highest sky in some submodel. The image of $a$ under (the canonical extension of) any finite-to-one function $\omega \rightarrow \omega$ is in the same sky as $a$. Conversely $[4,17]$, any two elements in the same sky have a common image via a finite-to-one function. Two ultrafilters are cofinally equivalent if and only if they occur as the types of two elements in the same sky of some model of arithmetic. This is the same as saying that the corresponding ultrapowers of $\omega$ can 
both be cofinally embedded in a single model; it is also equivalent to saying that these ultrapowers have cofinal submodels that are isomorphic [6].

An ultrafilter $U$ is a $Q$-point if and only if the highest sky in $U$-prod $\omega$ is a single constellation. An equivalent condition is that, if $a$ has type $U$ in a model $M$, then every $f(a)$, with $f$ standard, in the sky of $a$ is in fact in the constellation of $a$. It follows that, for such $a$ and $f$, the type $f(U)$ of $f(a)$ is isomorphic to $U$ and therefore also a $Q$-point.

LEMma 2.7. If $U$ is a $Q$-point, then no sky contains two distinct elements of type $U$.

Proof. Suppose $a, b$ were a counterexample. By the result from $[4,17]$ quoted above, since $a$ and $b$ are in the same sky, there would be a finite-to-one $f: \omega \rightarrow \omega$ such that $f(a)=f(b)$. As $U$ is a $Q$-point, there is a bijection $g: \omega \rightarrow \omega$ that agrees with $f$ on a set in $U$. It follows that $g(a)=f(a)=f(b)=g(b)$, and therefore $a=g^{-1}(g(a))=g^{-1}(g(b))=b$.

If two models $M_{1}$ and $M_{2}$ have isomorphic submodels $M_{1}^{\prime} \cong M_{2}^{\prime}$, then they can both be embedded in a third model in such a way that $M_{1}^{\prime}$ and $M_{2}^{\prime}$ are identified (along any prescribed isomorphism). In particular, if $a_{1} \in M_{1}$ and $a_{2} \in M_{2}$ have the same type, then $M_{1}$ and $M_{2}$ can be embedded in another model so that $a_{1}$ and $a_{2}$ have the same image.

If $\Phi$ is any set of first-order formulas with, say, free variables $x$ and $y$, then the compactness theorem provides a model of arithmetic containing two elements $a, b$ that satisfy all these formulas (one says that they realize the (partial) type $\Phi$ ) unless there is a finite subset of $\Phi$ that cannot be simultaneously satisfied on $\omega$. More generally, suppose $\Phi$ and $\Psi$ are sets of formulas with $x, y$ free, such that whenever $\Phi$ is satisfied by two elements in any model of arithmetic then $\Psi$ is also satisfied in that model by the same two elements; then each of the formulas $\psi(x, y) \in \Psi$ is a consequence, in $\omega$, of finitely many formulas $\varphi(x, y) \in \Phi$. (The finite set of $\varphi$ 's can depend on $\psi$.) A typical example of a set of formulas to which such considerations might be applied is the set "saying" that $x$ has type $U, y$ has type $V$, and $x$ is in a lower sky than $y$; this set consists of all formulas of the forms $x \in A, y \in B$, and $f(x)<y$, where $A$ ranges over $U, B$ over $V$, and $f$ over the set of all functions $\omega \rightarrow \omega$.

3. Decomposition of operator ideals. Let $\mathscr{H}$ be an infinite-dimensional, separable, complex Hilbert space, and let $\mathscr{L}$ be the algebra of bounded linear operators on $\mathscr{H}$. We shall be concerned with proper two-sided ideals in this algebra, called simply ideals in this section. Among these ideals, there is a largest one, $\mathscr{K}$, consisting of the compact operators. There is also a smallest nonzero ideal, $\mathscr{F}$, consisting of all operators of finite rank. Various sorts of intermediate ideals have been extensively studied; see for example [21]. Brown, Pearcy, and Salinas [9] asked whether $\mathscr{K}$ is the sum of two properly smaller ideals.

It was proved in [5] that the continuum hypothesis implies an affirmative answer to this question and, indeed, implies that every ideal that properly includes $\mathscr{F}$ is the sum of two properly smaller ideals. It was also stated in [5], but with only a hint 
toward a proof, that the continuum hypothesis can be replaced with (what in our present terminology is) the negation of NCF (see (**) on p. 414 of [5]). The purpose of the present section is to prove this result and its converse.

THEOREM 3.1. The following are equivalent.

(a) $\mathscr{K}$ is not the sum of two properly smaller ideals.

(b) Some ideal that properly includes $\mathscr{F}$ is not the sum of two properly smaller ideals.

(c) NCF.

Of course, (a) $\Rightarrow$ (b) is trivial. The nontrivial implications (b) $\Rightarrow$ (c) $\Rightarrow$ (a) both use the representation developed in [5] of ideals in terms of certain sets of sequences of natural numbers. We therefore begin by reviewing those aspects of the representation that we shall need.

Recall that $\omega \nearrow \omega$ is the set of nondecreasing sequences of natural numbers, viewed as functions $\omega \rightarrow \omega$. A nonempty subset $I$ of $\omega \nearrow \omega$ will be called a shift-ideal if it satisfies the following conditions.

(i) If $f \in I$ and $g \in \omega \nearrow \omega$ and $g(n) \leqslant f(n)$ for all $n$, then $g \in I$.

(ii) If $f \in I$ and $g \in I$, then $f+g \in I$.

(iii) If $f \in I$ and $g(n)=f(n+1)$ for all $n$, then $g \in I$.

In (iii), $g$ is $f$ shifted one step to the left; hence the name "shift-ideal." It is proved in the first part of [5] that there is a canonical isomorphism between the lattice of ideals of $\mathscr{L}$ and the lattice of shift-ideals. The ideal $\mathscr{K}$ corresponds to the shift-ideal $\omega \nearrow \omega$, while the ideal $\mathscr{F}$ corresponds to the shift-ideal of all bounded sequences. (Although we shall not need it, we indicate how the isomorphism is defined. Given an ideal, associate to each positive selfadjoint operator $A$ in it the sequence $f \in \omega \nearrow \omega$, where $f(n)$ is the number of eigenvalues $\geqslant 1 / 2^{n}$ of $A$, counted with multiplicity. The sequences so obtained from all the positive selfadjoint operators in the ideal constitute the corresponding shift-ideal.) The correspondence with shiftideals makes it easy to verify the known fact that the lattice of ideals of $\mathscr{L}$ is distributive.

In view of this representation of the lattice of ideals, Theorem 3.1 can be reformulated as follows; this is the form we shall prove.

THEOREM 3.2. The following are equivalent.

(a) $\omega \nearrow \omega$ is not the sum of two properly smaller shift-ideals.

(b) Some shift-ideal that contains an unbounded sequence is not the sum of two properly smaller shift-ideals.

(c) $N C F$.

Proof. (a) $\Rightarrow$ (b) is trivial.

(b) $\Rightarrow$ (c). We begin by recalling the relevant part of the proof in [5] that the continuum hypothesis implies the negation of (b). If $n \in \omega$ and if $A$ is any infinite subset of $\omega$ then $\operatorname{next}(A, n)$ was defined in $\S 2$ to be the smallest element of $A$ that is $\geqslant n$. The first part of the proof in [5] used the continuum hypothesis to construct two families, $\mathscr{B}_{1}$ and $\mathscr{B}_{2}$ of infinite subsets of $\omega$ such that

(i) If $X$ and $Y$ belong to the same $\mathscr{B}_{i}$, then some $Z$ in that $\mathscr{B}_{i}$ is almost included (i.e., included except for a finite subset) in both $X$ and $Y$. 
(ii) For every $f \in \omega \nearrow \omega$, there exist $X_{1} \in \mathscr{B}_{1}$ and $X_{2} \in \mathscr{B}_{2}$ such that, for all $n \in \omega$,

$$
f(n) \leqslant \max \left\{\operatorname{next}\left(X_{1}, n\right), \operatorname{next}\left(X_{2}, n\right)\right\} .
$$

(Actually, (i) was established in the stronger form that, if $X$ and $Y$ belong to the same $\mathscr{B}_{i}$, then one of $X$ and $Y$ is almost included in the other. However, only the weaker form stated above was actually used.) The second part of the proof used $\mathscr{B}_{1}$ and $\mathscr{B}_{2}$ to contradict (b) as follows. Let $I$ be a shift-ideal containing an unbounded $g \in \omega \nearrow \omega$. Let $h \in \omega \nearrow \omega$ be unbounded yet so small that, for any $a, b, c \in \omega$,

$$
a h(n+c)+b<g(n)
$$

for all but finitely many $n$. (Such an $h$ is easily constructed by diagonalization; see the lemma on p. 410 of [5].) Let $J_{i}^{\prime}$ for $i=1,2$ be the set of all $f \in \omega \nearrow \omega$ which are majorized by a linear function of $h$ on some set $X \in \mathscr{B}_{i}$, i.e., for all $n \in X$, $f(n) \leqslant a h(n)+b$. Let $J_{i}$ be the closure of $J_{i}^{\prime}$ under leftward shifting; thus, $f \in J_{i}$ if and only if for some $a, b, c \in \omega$

$$
f(n-c) \leqslant a h(n)+b
$$

for all $n$ in some set $X \in \mathscr{B}_{i}$. Then each $J_{i}$ is a shift-ideal not containing $g$, and, thanks to (ii), $J_{1}+J_{2}=\omega \nearrow \omega$. Therefore, the shift-ideals $J_{i} \cap I$ are properly included in $I$, and their sum is, by distributivity of the lattice, $I$. Thus, (b) fails.

To prove $(b) \Rightarrow(c)$, it therefore suffices to produce, under the assumption that NCF fails, two families $\mathscr{B}_{1}$ and $\mathscr{B}_{2}$ as above; then (b) will also fail, by the same proof as in [5]. The failure of NCF means that there exist two ultrafilters on $\omega$ with no common finite-to-one image. We take these ultrafilters as $\mathscr{B}_{1}$ and $\mathscr{B}_{2}$. Then (i) is clearly satisfied, and it remains only to prove (ii).

So let an arbitrary $f \in \omega \nearrow \omega$ be given. By Lemma 2.1, partition $\omega$ into blocks so that any natural number $n$ and its image $f(n)$ are in the same block or adjacent blocks. By Lemma 2.5, there exist $X_{1} \in \mathscr{B}_{1}$ and $X_{2} \in \mathscr{B}_{2}$ such that no union of two adjacent blocks meets both $X_{1}$ and $X_{2}$. Consider an arbitrary $n \in \omega$. The union $U$ of its block and the next is disjoint from $X_{i}$ for at least one of the two values of $i$. This means that $\operatorname{next}\left(X_{i}, n\right)>\max (U)$. Therefore,

$$
\max \left\{\operatorname{next}\left(X_{1}, n\right), \operatorname{next}\left(X_{2}, n\right)\right\}>\max (U) \geqslant f(n),
$$

where the second inequality comes from the specification of the blocks. This completes the verification of (ii) and thus the proof of $(b) \Rightarrow$ (c).

(c) $\Rightarrow$ (a). Assume NCF, and let $J_{1}$ and $J_{2}$ be shift-ideals properly included in $\omega \nearrow \omega$. Our goal is to show that $J_{1}+J_{2} \neq \omega \nearrow \omega$. This is obvious if one of the $J_{i}$ is $\{0\}$ (where 0 means the constant function with value 0 ), so we assume that neither $J_{i}$ is $\{0\}$. This means that each $J_{i}$ contains a function that is eventually positive (as the functions in $J_{i}$ are nondecreasing), hence a function that is everywhere positive (by shift closure), hence all bounded functions (by closure under addition and downward closure). Another application of closure under addition and downward closure of $J_{i}$ now shows that, if $f \in J_{i}$ and $g$ differs from $f$ at only finitely many places, then $g \in J_{i}$; we refer to this property of $J_{i}$ as closure under finite changes. 
As each $J_{i}$ is properly included in $\omega \nearrow \omega$, let $g \in(\omega \nearrow \omega)-J_{i}$. We can assume that $g$ is the same for both values of $i$, as two different $g$ 's could be replaced by their maximum (as the $J_{i}$ 's are closed downward). For each $f \in \omega \nearrow \omega$, let

$$
X_{f}=\{n \in \omega \mid f(n)<g(n)\} .
$$

For $f \in J_{i}$, the set $X_{f}$ is nonempty, as otherwise downward closure would require $J_{i}$ to contain $g$. Furthermore,

$$
X_{f} \cap X_{f^{\prime}}=X_{\max \left(f, f^{\prime}\right)} \supseteq X_{f+f^{\prime}}
$$

As $J_{i}$ is closed under addition, the sets $X_{f}$ for $f \in J_{i}$ generate a filter $F_{i}$. As $J_{i}$ is closed under finite changes, each of the two filters $F_{i}$ includes all cofinite subsets of $\omega$. By NCF, these two filters nearly cohere; that is, $\omega$ can be partitioned into blocks of consecutive integers so that each set in $F_{1}$ and each set in $F_{2}$ both meet some block (see Lemma 2.6).

Apply this to the sets $X_{f_{1}}$ and $X_{f_{2}}$, where $f_{1}$ and $f_{2}$ are arbitrary members of $J_{1}$ and $J_{2}$, respectively. There exist $m_{1} \in X_{f_{1}}$ and $m_{2} \in X_{f_{2}}$ such that $m_{1}$ and $m_{2}$ are in the same block. Suppose, without loss of generality, that $m_{1} \leqslant m_{2}$. Then

$$
f_{1}\left(m_{1}\right)<g\left(m_{1}\right) \leqslant g\left(m_{2}\right),
$$

as $m_{1} \in X_{f_{1}}$ and $g$ is nondecreasing, and

$$
f_{2}\left(m_{1}\right) \leqslant f_{2}\left(m_{2}\right)<g\left(m_{2}\right),
$$

as $f_{2}$ is nondecreasing and $m_{2} \in X_{f_{2}}$. Thus, if we define

$$
h(n)=2 \cdot g(\text { the last element of the block containing } n),
$$

then adding the two inequalities above yields, as $g$ is nondecreasing and $m_{1}$ and $m_{2}$ are in the same block,

$$
f_{1}\left(m_{1}\right)+f_{2}\left(m_{1}\right)<2 g\left(m_{2}\right) \leqslant h\left(m_{1}\right) .
$$

Since the $f_{i}$ 's were arbitrary members of the $J_{i}$ 's, this proves that $h \notin J_{1}+J_{2}$, so $J_{1}+J_{2} \neq \omega \nearrow \omega$

In view of the independence of NCF [7], we immediately obtain a "solution" of the question posed by Brown, Pearcy, and Salinas.

COROLlaRy 3.3. Whether $\mathscr{K}$ is the sum of two properly smaller ideals is independent of $Z F C$.

4. The Stone-Čech remainder of a half-line. Let $\mathbf{R}^{+}$be the closed half-line $[0, \infty)$ and let $\beta \mathbf{R}^{+}$be its Stone-Čech compactification. We shall be concerned with the remainder, that is, the part at infinity, $\beta \mathbf{R}^{+}-\mathbf{R}^{+}$, of this compactification. It is not hard to verify that $\beta \mathbf{R}^{+}-\mathbf{R}^{+}$is a continuum, i.e., a compact connected Hausdorff space. Bellamy [2] proved that it is indecomposable, which means that it is not the union of two proper subcontinua. On any indecomposable continuum, the binary relation "there is a proper subcontinuum containing both $x$ and $y$ " is an equivalence relation (indecomposability being used to ensure transitivity), and its equivalence classes are called the composants of the continuum. Metrizable indecomposable continua always have uncountably many composants, but Bellamy [2] showed that 
$\beta \mathbf{R}^{+}-\mathbf{R}^{+}$is not metrizable; he asked how many composants it has and in particular whether it might be an example of an indecomposable continuum with only one composant. (He later found a different example [3].) Rudin [19] showed, however, that the continuum hypothesis implies that $\beta \mathbf{R}^{+}-\mathbf{R}^{+}$has $2^{\aleph_{1}}$ composants.

Her proof shows, in fact, that cofinally inequivalent ultrafilters, considered as points of $\beta \omega \subseteq \beta \mathbf{R}^{+}$, are in different composants of $\beta \mathbf{R}^{+}-\mathbf{R}^{+}$. (This was pointed out to me by van Douwen.) In particular, if $\beta \mathbf{R}^{+}-\mathbf{R}^{+}$has only one composant, then NCF holds. The converse of this was announced by Mioduszewski [15]; it is easily deduced from his detailed results in [16] on the structure of $\beta \mathbf{R}^{+}$, though it is not explicitly stated in [16]. In this section, we give a proof of this equivalence between NCF and the existence of only one composant in $\beta \mathbf{R}^{+}-\mathbf{R}^{+}$. The proof is based on the same construction as Bellamy's proof of indecomposability. In one direction it is essentially the same as Rudin's proof; in the other direction, it seems a bit simpler than Mioduszewski's, because it is directed specifically toward this one result whereas Mioduszewski develops a general structure theory for $\beta \mathbf{R}^{+}$.

THEOREM 4.1 (Rudin, Mioduszewski). NCF is equivalent to the assertion that $\beta \mathbf{R}^{+}-\mathbf{R}^{+}$has only one composant.

Proof. We begin by considering an arbitrary proper subcontinuum $K$ of $\beta \mathbf{R}^{+}-$ $\mathbf{R}^{+}$. Let $p$ be a point of $\left(\beta \mathbf{R}^{+}-\mathbf{R}^{+}\right)-K$, and let $G$ and $H$ be open neighborhoods of $K$ and $p$ with disjoint closures in $\beta \mathbf{R}^{+}$; such neighborhoods exist since $\beta \mathbf{R}^{+}$is a compact Hausdorff space. Since $\mathbf{R}^{+}$is dense in $\beta \mathbf{R}^{+}, G \cap \mathbf{R}^{+}$is dense in $G$, and similarly for $H$. In particular, each of $G \cap \mathbf{R}^{+}$and $H \cap \mathbf{R}^{+}$contains arbitrarily large real numbers. Following Bellamy [2], we inductively define real numbers $p_{n}<q_{n}<$ $r_{n}<s_{n}<p_{n+1}$, for all $n \in \omega$, by

$$
\begin{aligned}
& p_{n}=\inf \left(G \cap\left[s_{n-1}, \infty\right)\right) \quad\left(\text { where }\left[s_{-1}, \infty\right) \text { means } \mathbf{R}^{+}\right), \\
& q_{n}=\sup \left\{x \in G \mid x>p_{n} \text { and }\left[p_{n}, x\right] \text { is disjoint from } H\right\}, \\
& r_{n}=\inf \left(H \cap\left[q_{n}, \infty\right)\right), \text { and } \\
& s_{n}=\sup \left\{x \in H \mid x>r_{n} \text { and }\left[r_{n}, x\right] \text { is disjoint from } G\right\} .
\end{aligned}
$$

Because the closures $\bar{G}$ and $\bar{H}$ are disjoint, it is easy to see that these numbers satisfy the inequalities asserted above. The $p$ 's and $q$ 's are in $\bar{G}$ while the $r$ 's and $s$ 's are in $\bar{H}$. It follows that the sequence of $p$ 's, $q$ 's, $r$ 's, and $s$ 's must tend to infinity, for otherwise its limit would be in both $\bar{G}$ and $\bar{H}$ which are disjoint. From this it follows easily that the intervals $\left[p_{n}, q_{n}\right]$ cover $G \cap \mathbf{R}^{+}$and that the intervals $\left[r_{n}, s_{n}\right]$ cover $H \cap \mathbf{R}^{+}$except for $H \cap\left[0, p_{0}\right]$. We remove this initial segment from $H$ and assume henceforth that the intervals $\left[r_{n}, s_{n}\right]$ cover $H \cap \mathbf{R}^{+}$.

Let us write $I_{2 n}$ for $\left[p_{n}, q_{n}\right], I_{2 n+1}$ for $\left[r_{n}, s_{n}\right]$, and $I_{X}$ for $\bigcup_{n \in X} I_{n}$, where $X \subseteq \omega$. Thus, for example, $G \cap \mathbf{R}^{+} \subseteq I_{\text {Even }}$, where "Even" stands for the set of even numbers. Let $U=\left\{X \subseteq \omega \mid K \subseteq \overline{I_{X}}\right\}$, where the closure of $I_{X}$ is taken in $\beta \mathbf{R}^{+}$. Since $I_{\text {Even }}$ includes $G \cap \mathbf{R}^{+}$, which is dense in $G$, which includes $K$, the set Even is in $U$. $U$ is clearly closed under supersets. It does not contain two disjoint sets, for if $X$ and $Y$ are disjoint then $I_{X}$ and $I_{Y}$ are disjoint closed sets in $\mathbf{R}^{+}$, so their closures in $\beta \mathbf{R}^{+}$ 
are also disjoint, by a basic property of Stone-Čech compactifications [11, Theorem 6.5]. If $U$ contains the union of two disjoint sets, then it contains one of those sets, because $\overline{I_{X \cup Y}}=\overline{I_{X} \cup I_{Y}}=\overline{I_{X}} \cup \overline{I_{Y}}$ and, if the connected set $K$ lies in the union of the disjoint closed sets $\overline{I_{X}}$ and $\overline{I_{Y}}$, then it lies in one of them. $U$ contains no finite set, for when $X$ is finite then $I_{X}$ is compact and $\overline{I_{X}}=I_{X}$ is disjoint from $\beta \mathbf{R}^{+}-\mathbf{R}^{+}$. According to Lemma 2.2, the preceding five sentences establish that $U$ is an ultrafilter. Thus, we have shown that any proper subcontinuum $K$ of $\beta \mathbf{R}^{+}-\mathbf{R}^{+}$is included in $\bigcap_{X \in U} \overline{I_{X}}=\bigcap_{X \in U} \overline{\bigcup_{n \in X} I_{n}}$ for some sequence of disjoint closed intervals $I_{N} \subseteq \mathbf{R}^{+}$and some ultrafilter $U$.

Suppose now that $K$ contains a point $v \in \beta \omega-\omega \subseteq \beta \mathbf{R}^{+}-\mathbf{R}^{+}$; we can identify $v$ with an ultrafilter $V$ on $\omega$. Let $f: \omega \rightarrow \omega$ be a finite-to-one function whose value on $\omega \cap I_{n}$ is $n$. (It does not matter how $f$ is defined on $\omega-I_{n}$ as long as it is finite-to-one.) Then, for each $X \in U$ and each $Y \in V$ we have that $v$ lies in both $\overline{I_{X}}$ and $\bar{Y}$. Since disjoint closed sets in $\mathbf{R}^{+}$have disjoint closures in $\beta \mathbf{R}^{+}$, the closed sets $I_{X}$ and $Y$ cannot be disjoint. This means that $f(Y)$ meets $X$. Since $X$ was an arbitrary set in $U$ and since $U$ is an ultrafilter, it follows that $f(Y) \in U$. Since $Y$ was an arbitrary set in $V$, it follows that $f(V)=U$.

If $v^{\prime}$ is a second point of $(\beta \omega-\omega) \cap K$, identified with an ultrafilter $V^{\prime}$ on $\omega$, then $V$ and $V^{\prime}$ are cofinally equivalent, because $U$ is a finite-to-one image of each of them. Since $K$ was an arbitrary proper subcontinuum of $\beta \mathbf{R}^{+}-\mathbf{R}^{+}$, we see that any two ultrafilters that, viewed as points of $\beta \mathbf{R}^{+}-\mathbf{R}^{+}$, lie in the same composant, must be cofinally equivalent. In particular, if there is only one composant, then all ultrafilters are cofinally equivalent, which means that NCF holds.

To prove the converse, assume that NCF holds, and let $p$ and $q$ be two distinct points in $\beta \mathbf{R}^{+}-\mathbf{R}^{+}$. Applying the first part of this proof with $\{q\}$ as $K$ (and with $p$ as $p$ ), we find a sequence of disjoint closed intervals $I_{n}$, where $I_{n+1}$ always lies to the right of $I_{n}$, such that $q \in \bigcap_{X \in U} \overline{I_{X}}$ and similarly $p \in \bigcap_{X \in V} \overline{I_{X}}$ for suitable ultrafilters $U$ and $V$ (with Even $\in U$ and Odd $\in V$ ). By NCF, let $f: \omega \rightarrow \omega$ be a finite-to-one nondecreasing function such that $f(U)=f(V)=W$, for a certain ultrafilter $W$. For each $n$, let $J_{n}$ be the convex hull of $I_{f^{-1}\{n\}}$. Thus, each $J_{n}$ is a closed interval (since $f$ is finite-to-one), and $J_{n+1}$ lies strictly to the right of $J_{n}$ (since $f$ is nondecreasing). Let $J_{X}=\bigcup_{n \in X} J_{n}$, and observe that, for any $X \subseteq \omega, I_{X} \subseteq J_{f(X)}$. As $X$ ranges over $U$ (or $V$ ), $f(X)$ ranges over a basis for $W$ (in fact all of $W$ if we take $f$ to map onto $\omega$ ). Thus,

$$
q \in \bigcap_{X \in U} \overline{I_{X}} \subseteq \bigcap_{X \in U} \overline{J_{f(X)}}=\bigcap_{Y \in W} \overline{J_{Y}}
$$

and similarly $p \in \bigcap_{Y \in W} \overline{J_{Y}}$. Thus, to prove that $p$ and $q$ lie in the same composant of $\beta \mathbf{R}^{+}-\mathbf{R}^{+}$, it suffices to show that $\bigcap_{Y \in W} \overline{J_{Y}}$ is a proper subcontinuum of $\beta \mathbf{R}^{+}-\mathbf{R}^{+}$. It is obviously compact. It is included in $\beta \mathbf{R}^{+}-\mathbf{R}^{+}$because $W$ is nonprincipal and the intervals $J_{n}$ tend to infinity (as the $I_{n}$ 's did). It is a proper subset of $\beta \mathbf{R}^{+}-\mathbf{R}^{+}$because it is disjoint from $\bar{J}_{Z}$ for any $Z \notin W$, and $\bar{J}_{Z}$ meets $\beta \mathbf{R}^{+}-\mathbf{R}^{+}$whenever $Z$ is infinite. All that remains is to prove that $\bigcap_{Y \in W} \overline{J_{Y}}$ is connected. 
Suppose it were not, and partition it into two disjoint nonempty closed subsets $C_{0}$ and $C_{1}$. Being closed also in $\beta \mathbf{R}^{+}$, these sets have disjoint neighborhoods $G_{0}$ and $G_{1}$ in $\beta \mathbf{R}^{+}$. The closed sets $\overline{J_{X}}-\left(G_{0} \cup G_{1}\right)$ for $X \in W$ cannot all be nonempty, for then, as they form a directed family, compactness would make their intersection $\left(\bigcap_{X \in W} \overline{J_{X}}\right)-\left(G_{0} \cup G_{1}\right)$ nonempty, contrary to our choice of the $C$ 's and $G$ 's. So fix an $X \in W$ with $\overline{J_{X}} \subseteq G_{0} \cup G_{1}$. Each of the intervals $J_{k} \subseteq \overline{J_{X}}$, for $k \in X$, must lie entirely within one of the two $G_{i}$ 's because $J_{k}$ is connected. As $W$ is an ultrafilter, it contains the set

$$
Y_{i}=\left\{k \in X \mid J_{k} \subseteq G_{i}\right\}
$$

for one of the two values of $i$; fix this $i$ and write simply $Y$ for $Y_{i}$. Then $J_{Y} \subseteq G_{i}$, so

$$
\bigcap_{X \in W} \overline{J_{X}} \subseteq \overline{J_{Y}} \subseteq \overline{G_{i}}
$$

But this is absurd, because $C_{1-i}$ is a nonempty subset of $\bigcap_{X \in W} \overline{J_{X}}$ disjoint from $\bar{G}_{i}$. This contradiction completes the proof that $\bigcap_{X \in W} \overline{J_{X}}$ is a proper subcontinuum of $\beta \mathbf{R}^{+}-\mathbf{R}^{+}$and thus completes the proof of the theorem.

The theorem allows us to apply the consistency proof from [7].

CoROllaRY 4.2. It is consistent, relative to $Z F C$, that $\beta \mathbf{R}^{+}-\mathbf{R}^{+}$has only one composant.

5. Ideals in the lattice $\omega \nearrow \omega$. A nonempty subset $I$ of $\omega \nearrow \omega$ is an ideal if it is closed downward and closed under binary maxima. That is,

(i) if $f \in I$ and $g(n) \leqslant f(n)$ for all $n$, then $g \in I$, and

(ii) if $f, g \in I$, then $\max (f, g) \in I$,

where $\max (f, g)(n)=\max \{f(n), g(n)\}$ for all $n$. In this section, we study a certain pre-ordering on the class of ideals in $\omega \nearrow \omega$. This ordering, restricted to a certain subclass of the ideals, was introduced for group-theoretic purposes in [12]. Consideration of all ideals, rather than a restricted class, seems more natural from a combinatorial point of view and simplifies some of the work. To obtain information relevant to the group-theoretic study in [12], one must pass to the restricted class, and this will be done in the next section.

If $I$ and $J$ are two ideals in $\omega \nearrow \omega$, we define $I \leqslant J$ to mean that there exists $r \in \omega \nearrow \omega$ such that, for each $f \in I$, there exists $g \in J$ with $f(n) \leqslant g(r(n))$ for all sufficiently large $n$. That is, if all the functions in $J$ are composed on the right with $r$, then each function in $I$ is eventually majorized by one in the modified $J$. Notice that, if $r$ is as required by the definition of $I \leqslant J$, then so is any $r^{\prime}$ that eventually majorizes $r$.

The following lemma says that, instead of modifying $J$, we could have modified $I$ by composing with a sort of inverse to $r$.

LEMMA 5.1. Let $I$ and $J$ be ideals in $\omega \nearrow \omega$. Then $I \leqslant J$ if and only if there exists an unbounded $s \in \omega \nearrow \omega$ such that, for each $f \in I$, there exists $g \in J$ with $f(s(n)) \leqslant g(n)$ for all sufficiently large $n$. 
PROOF. For "if," use the fact that $s$ is unbounded and nondecreasing to define $r(n)$ as the first integer greater than every $k$ for which $s(k)<n$. Then $r \in \omega \nearrow \omega, r$ is unbounded, and $s(r(n)) \geqslant n$ for all $n$. Thus, if $f(s(n)) \leqslant g(n)$ for all large $n$, then

$$
f(n) \leqslant f(s(r(n))) \leqslant g(r(n))
$$

for all large $n$. For the converse, define $s(n)$ to be the largest $k$ for which $r(k) \leqslant n$; if there is no such $k$, i.e., if $n<r(0)$, set $s(n)=0$. Then $s \in \omega \nearrow \omega$, $s$ is unbounded, and $r(s(n)) \leqslant n$ for all $n \geqslant r(0)$. Thus, if $f(n) \leqslant g(r(n))$ for all large $n$, then $f(s(n)) \leqslant g(r(s(n))) \leqslant g(n)$ for all large $n$.

It is clear that the relation $\leqslant$ on ideals is reflexive (take $r=$ identity) and transitive (compose the $r$ 's). It therefore induces an equivalence relation on the ideals and a partial ordering of the equivalence classes. We shall sometimes abuse language by referring to ideals when we mean equivalence classes; for example, we may refer to the partial ordering of ideals. This purpose of this section is to present a connection between NCF and the structure of this ordering. Although the connection involves phenomena near the top of the ordering, we first give, for the sake of completeness, some elementary facts about the bottom.

Define the ideals

$$
\begin{aligned}
& B_{k}=\{f \in \omega \nearrow \omega \mid \text { for all } n, f(n) \leqslant k\}, \text { for each } k \in \omega, \\
& B=\{f \in \omega \nearrow \omega \mid f \text { is bounded }\}=\bigcup_{k \in \omega} B_{k}, \text { and } \\
& L=\{f \in \omega \nearrow \omega \mid \text { for all } n, f(n) \leqslant n\} .
\end{aligned}
$$

It is easy to compute, directly from the definition of $\leqslant$, that for every ideal $I$,

$$
\begin{aligned}
& B_{0} \leqslant I, \\
& B_{k+1} \leqslant I \Leftrightarrow I \nsubseteq B_{k}, \\
& I \leqslant B_{k} \Leftrightarrow I \subseteq B_{k}, \\
& B \leqslant I \Leftrightarrow \text { for all } k, I \nsubseteq B_{k}, \\
& I \leqslant B \Leftrightarrow I \subseteq B, \\
& L \leqslant I \Leftrightarrow I \nsubseteq B, \text { and } \\
& I \leqslant L \Leftrightarrow I \text { is dominated, i.e. there exists } r \in \omega \nearrow \omega \text { such that, for } \\
& \quad \text { each } f \in I, f(n) \leqslant r(n) \text { for all sufficiently large } n .
\end{aligned}
$$

From these facts, it immediately follows that the bottom of the ordering of ideals is $B_{0}<B_{1}<\cdots<B_{k}<\cdots<B<L$, and that every ideal not equivalent to any of these is undominated and $>L$.

Turning to the top of the ordering, we have first the obvious greatest element, $\omega \nearrow \omega$. The ideals $I$ equivalent to this one are, as one easily verifies using Lemma 5.1 , those such that every $f \in \omega \nearrow \omega$ is eventually majorized by a member of $I$ and therefore eventually equal to a member of $I$.

The equivalence classes of ideals described in the last two paragraphs are the only ones that admit a simple description, in the following precise sense. If an ideal $I$ is a Borel subset, or even an analytic subset, of $\omega \nearrow \omega$ (with respect to the topology 
induced by the product topology on the product ${ }^{\omega} \omega$ of countably many copies of $\omega$, each copy having the discrete topology), then either $I \leqslant L$ or $I$ is equivalent to $\omega \nearrow \omega$. The proof, which we only sketch here because it is peripheral to our main subject, rests on the result of Kechris [14] and Saint-Raymond [20] that every analytic subset of $\omega \nearrow \omega$ (or equivalently ${ }^{\omega} \omega$ ) either is included in a $K_{\sigma}$ set or has a superperfect subset. A $K_{\sigma}$ set is precisely a dominated set, so if it is an ideal then it is $\leqslant L$. On the other hand, given any superperfect subset $S$ of $\omega \nearrow \omega$ and any $f \in \omega \nearrow \omega$, we can produce two elements $s$ and $t$ of $S$ such that $\max (s, t)$ eventually majorizes $f$. ( $s$ and $t$ are obtained by inductively defining increasing sequences of nodes $s_{n}$ and $t_{n}$, in the superperfect tree $T$ of finite sequences associated to $S$, so that each $s_{n}$ and each $t_{n}$ is a superbranching node, i.e., one with infinitely many immediate successors. Let $s_{0}$ be any superbranching node and $t_{0}$ any superbranching extension of it. If $s_{n}, t_{n}$ have been defined and have lengths $a$ and $b$, where $a<b$, then as $s_{n}$ is superbranching we can extend it to $s^{\prime} \in T$ with $s^{\prime}(a) \geqslant f(b)$ and then extend it further to a superbranching $s_{n+1}$, of length $c>b$. This ensures that $s \geqslant f$ on $[a, b]$. As $t_{n}$ is superbranching, extend it to $t^{\prime} \in T$ with $t(b) \geqslant f(c)$, and extend it further to a superbranching $t_{n+1}$ of length $d>c$. This ensures that $t \geqslant f$ on $[b, c]$.) It follows that any ideal that includes a superperfect $S$ eventually majorizes all functions, hence is equivalent to $\omega \nearrow \omega$. The assumption of analyticity can be weakened under suitable hypotheses (e.g. to projectivity if one assumes projective determinacy).

To obtain ideals not equivalent to those already listed, one therefore needs some highly nonconstructive approach. Göbel and Wald [13] showed (in the context of their restricted sort of ideals) that a nonprincipal ultrafilter on $\omega$ provides such an approach; it yields a new equivalence class of ideals. (I know of no essentially different way to obtain such ideals.)

For any nonprincipal ultrafilter $U$ on $\omega$, define

$$
I(U)=\{f \in \omega \nearrow \omega \mid \text { for some } A \in U, f \leqslant \text { identity on } A\} .
$$

This is clearly an ideal. It contains, for each $A \in U$, the function $\operatorname{next}(A,-)$ defined in $\S 2$, since this function equals the identity on $A$. For any $f \in I(U)$, if $A \in U$ is as in the definition of $I(U)$ then for all $n$

$$
f(n) \leqslant f(\operatorname{next}(A, n)) \leqslant \operatorname{next}(A, n),
$$

the first inequality being because $f$ is nondecreasing and the second because $\operatorname{next}(A, n)$ is in $A$ where $f \leqslant$ identity. This shows that $I(U)$ is the downward closure of the family of functions next $(A,-)$ where $A$ ranges over $U$.

The ideal $I(U)$ is not equivalent to any of those exhibited earlier. No function in it eventually majorizes $n \mapsto n+1$, so it is not equivalent to $\omega \nearrow \omega$. That it is not dominated and therefore not $\leqslant L$ is shown by the following argument from [22]. Suppose $r \in \omega \nearrow \omega$ eventually majorizes $\operatorname{next}(A,-)$ for all $A \in U$. Inductively define natural numbers $a_{0}, a_{1}, \ldots$ by setting $a_{0}=0$ and $a_{n+1}=1+$ $\max \left\{a_{n}, r\left(a_{n}\right)\right\}$. Then for any $A \in U$, for all sufficiently large $n$,

$$
\operatorname{next}\left(A, a_{n}\right) \leqslant r\left(a_{n}\right)<a_{n+1},
$$


so $A$ intersects all but finitely many of the intervals $\left[a_{n}, a_{n+1}\right)$. Thus, neither the set $\cup_{n \text { even }}\left[a_{n}, a_{n+1}\right)$ nor its complement is in $U$, which contradicts the fact that $U$ is an ultrafilter.

Thus, we have at least one ideal $I(U)$ (possibly many, if we vary $U$ ) strictly between $L$ and $\omega \nearrow \omega$. We next show that at least some of these ideals are high in the ordering.

Proposition 5.2. Every ideal $J<\omega \nearrow \omega$ is $\leqslant I(U)$ for some $U$.

Proof. Given $J<\omega \nearrow \omega$, fix a function $r \in \omega \nearrow \omega$ not eventually majorized by any element of $J$. Increasing $r$, if necessary, we assume that $r$ is strictly monotone and we record, for future reference, that this implies

$$
r(\operatorname{next}(A, n))=\operatorname{next}(r(A), r(n))
$$

for all $n \in \omega$ and all infinite $A \subseteq \omega$.

For each $f \in J$, the set

$$
M_{f}=\{n \in \omega \mid f(n)<r(n)\}
$$

is, by our choice of $r$, infinite. As $J$ is an ideal and $M_{f} \cap M_{g}=M_{\max (f, g)}$, we see that the sets $M_{f}$ for $f \in J$ generate a filter of infinite sets. Let $U^{\prime}$ be any ultrafilter extending this filter. Thus, each $f \in J$ is $<r$ on a set in $U^{\prime}$. We shall show that $U=r\left(U^{\prime}\right)$ is as required, i.e. that $J \leqslant I(U)$. The function $r$ required by the definition of $\leqslant$ will be the $r$ that we have already been using. To see that it works, consider any $f \in J$, and let $A=M_{f} \in U^{\prime}$. Then $r(A) \in r\left(U^{\prime}\right)=U$ and we have, by definition of $M_{f}$ and monotonicity of $f$, for all $n$,

$$
f(n) \leqslant f(\operatorname{next}(A, n))<r(\operatorname{next}(A, n))=\operatorname{next}(r(A), r(n))=g(r(n)),
$$

where $g=\operatorname{next}(r(A),-) \in I(U)$.

The $I(U)$ 's, as $U$ varies, are therefore cofinal in our ordering minus its top element. The remainder of this section is concerned primarily with the relative ordering of the various $I(U)$ 's. We shall obtain among other results, the following equivalents of $\mathrm{NCF}$.

THEOREM 5.3. The following are equivalent.

(a) $N C F$.

(b) The ideals $I(U)$ are all equivalent.

(c) The ideals $I(U)$ are pairwise comparable under $\leqslant$.

(d) The ordering of equivalence classes of ideals, minus its top element $\omega \nearrow \omega$, has a largest element.

The preceding proposition gives (b) $\Rightarrow(d)$, and of course (b) $\Rightarrow(c)$ is trivial. To prove the rest of the theorem, we develop a criterion, in terms of ultrapowers of $\omega$, for $I(U) \leqslant I(V)$.

THEOREM 5.4. Let $U$ and $V$ be ultrafilters. Then $I(U) \leqslant I(V)$ if and only if, in some model of arithmetic, there exist elements $u \leqslant v$, of types $U$ and $V$ respectively, and lying in the same sky. 
Proof. Since $I(U)$ is the downward closure of the functions $\operatorname{next}(A,-)$ for $A \in U$, and similarly for $V$, the definition of $I(U) \leqslant I(V)$ reduces to

$$
\begin{aligned}
& (\exists r \in \omega \nearrow \omega)(\forall A \in U)(\exists B \in V) \\
& (\forall \text { sufficiently large } n) \operatorname{next}(A, n) \leqslant \operatorname{next}(B, r(n)) .
\end{aligned}
$$

By removing finitely many elements from $B$, we can improve ( $\forall$ sufficiently large $n$ ) to $(\forall n)$, obtaining the equivalent form

$$
(\exists r \in \omega \nearrow \omega)(\forall A \in U)(\exists B \in V) B \text { is disjoint from } \bigcup_{n \in \omega}[r(n), \operatorname{next}(A, n)) .
$$

As $V$ is an ultrafilter, to say that it contains a set $B$ disjoint from a certain $X$ is the same as to say that every $B \in V$ contains points outside $X$. Thus, $I(U) \leqslant I(V)$ if and only if

$$
(\exists r \in \omega \nearrow \omega)(\forall A \in U)(\forall B \in V)(\exists b \in B) b \notin \bigcup_{n \in \omega}[r(n), \operatorname{next}(A, n)) .
$$

To put " $b \notin \bigcup_{n \in \omega}[r(n), \operatorname{next}(A, n))$ " into a more useful form, consider for a moment a fixed $b$, and let $a$ be the last element of $A$ that is $\leqslant b$. (We can ignore the finitely many $b$ 's for which no such $a$ exists.) Then clearly $b \notin[r(n), \operatorname{next}(A, n))$ for $n \leqslant a$, since then $\operatorname{next}(A, n) \leqslant a \leqslant b$. For $n>a$, however, we have $b<\operatorname{next}(A, n)$, so in order to have $b \notin[r(n), \operatorname{next}(A, n))$, we must have $b<r(n)$. Having this for all $n>a$ is equivalent, since $r$ is nondecreasing, to having it for $n=a+1$. Thus, $b \notin \bigcup_{n \in \omega}[r(n), \operatorname{next}(A, n))$ if and only if there exists $a \in A$ with $a \leqslant b<r(a+1)$. Writing $s(n)$ for $r(n+1)$, we have that $I(U) \leqslant I(V)$ if and only if

$$
(\exists s \in \omega \nearrow \omega)(\forall A \in U)(\forall B \in V)(\exists a \in A)(\exists b \in B) a \leqslant b<s(a) .
$$

This is equivalent to saying that, for some $s \in \omega \nearrow \omega$, the 2-type in arithmetic saying " $x$ has type $U, y$ has type $V$, and $x \leqslant y<s(x)$ " is consistent, hence has a model. Thus, $I(U) \leqslant I(V)$ if and only if, for some $s \in \omega \nearrow \omega$, there is a model of arithmetic containing elements $u$ of type $U$ and $v$ of type $V$ with $u \leqslant v<s(u)$.

COROLlaRY 5.5. $I(U)$ and $I(V)$ are comparable under $\leqslant$ if and only if $U$ and $V$ are cofinally equivalent.

Proof. By the theorem, $I(U)$ and $I(V)$ are $\leqslant$-comparable if and only if, in some model of arithmetic, there are elements of types $U$ and $V$ in the same sky. This sky may be assumed to be the top one, by truncating the model after it. Then the submodels generated by the two specified elements are both cofinal in the ambient model and are isomorphic to $U$-prod $\omega$ and $V$-prod $\omega$ respectively. So $U$ and $V$ are cofinally equivalent. The converse is proved by reversing these steps, using Corollary 2 of [6].

This corollary immediately gives us (a) $\Leftrightarrow$ (c) in Theorem 5.3. It also gives us (d) $\Rightarrow(a)$, as follows. Suppose that, among the equivalence classes of ideals other than that of $\omega \nearrow \omega$, there is a greatest one. By Proposition 5.2, this greatest equivalence class must contain $I(U)$ for some ultrafilter $U$. Now the preceding corollary tells us that all ultrafilters are cofinally equivalent to $U$, hence to each other. 
The parts of Theorem 5.3 established so far can be summarized as (b) $\Rightarrow$ (d) $\Rightarrow$ (a) $\Leftrightarrow$ (c). The proof will be completed if we prove (a) $\Rightarrow$ (b).

The preceding corollary tells us that the ideals $I(U)$ are ordered by $\leqslant$ as a disjoint union of chains, namely the cofinal equivalence classes, such that elements of different chains are incomparable. To complete the analysis of the ordering of the $I(U)$ 's, we study the chains individually. They turn out to look quite different, depending on whether or not the corresponding cofinal equivalence class of ultrafilters contains a $Q$-point. We begin with the case that it does not, since this is the case that is relevant to Theorem 5.3.

Proposition 5.6. If $U$ and $V$ are cofinally equivalent ultrafilters whose cofinalequivalence class contains no $Q$-point, then $I(U)$ is equivalent to $I(V)$.

Proof. Suppose $U$ and $V$ form a counterexample. As they are cofinally equivalent, there is a model $M$ of arithmetic containing elements $u$ and $v$, of types $U$ and $V$ respectively, in the same sky. Assume without loss of generality that $u \leqslant v$. By Theorem 5.4, $I(U) \leqslant I(V)$. But $I(U)$ and $I(V)$ are assumed to be inequivalent, so $I(V) \nless I(U)$. Thus, there is no model $M^{\prime}$ of arithmetic with two elements $u^{\prime}$ and $v^{\prime}$, of types $U$ and $V$ respectively, in the same sky, with $v^{\prime} \leqslant u^{\prime}$.

Since $u$ and $v$ are in the same sky, fix an $s: \omega \rightarrow \omega$ such that $v \leqslant s(u)$. If there were a model $M^{\prime \prime}$ of arithmetic containing two elements $u_{1}$ and $u_{2}$, both of type $U$, in the same sky, with $s\left(u_{1}\right) \leqslant u_{2}$, then we could amalgamate it with $M$, identifying $u \in M$ with $u_{1} \in M^{\prime \prime}$, obtaining an $M^{\prime}$ containing $u_{2}$ and $v$, of types $U$ and $V$ respectively, in the same sky (as both are in the sky of $u_{1}=u$ ), with $v \leqslant s(u)=$ $s\left(u_{1}\right) \leqslant u_{2}$. By the preceding paragraph, no such $M^{\prime}$ exists; therefore no such $M^{\prime \prime}$ exists.

This means that, whenever the 2-type saying " $x_{1}$ and $x_{2}$ both have type $U$ and $s\left(x_{1}\right) \leqslant x_{2}$ " is realized by $u_{1}$ and $u_{2}$ in a model of arithmetic, so is the type saying " $x_{2}$ is in a higher sky than $x_{1}$." Thus, every formula in the latter type must be a consequence in arithmetic of the former type. In other words,

$$
(\forall f: \omega \rightarrow \omega)(\exists A \in U)\left(\forall a_{1}, a_{2} \in A\right)\left(s\left(a_{1}\right) \leqslant a_{2} \Rightarrow f\left(a_{1}\right)<a_{2}\right) .
$$

Partition $\omega$ into blocks so that, for any $n, s(n)$ is in the next block after $n$, or earlier. Thus, if $a_{1}<a_{2}$ are not in the same block and not in adjacent blocks, then $s\left(a_{1}\right)<a_{2}$. Thus,

$$
(\forall f: \omega \rightarrow \omega)(\exists A \in U)\left(\forall a_{1}<a_{2} \text { in } A\right)
$$

If $a_{1}$ and $a_{2}$ are not in the same block or adjacent blocks, then $f\left(a_{1}\right)<a_{2}$.

Define $h: \omega \rightarrow \omega$ to map all elements of the $n$th block to $n$, for all $n \in \omega$. Let $g$ be any finite-to-one function on $\omega$ (viewed as the range of $h$ ). Apply the result of the preceding paragraph with

$$
f(n)=\text { the largest } k \text { such that } g(h(k))=g(h(n)),
$$

and let $A \in U$ be as there. Thus, if $a_{1}<a_{2}$ are in $A$ and $h\left(a_{1}\right)$ differs from $h\left(a_{2}\right)$ by more than 1 , then $f\left(a_{1}\right)<a_{2}$, which means that $g\left(h\left(a_{1}\right)\right) \neq g\left(h\left(a_{2}\right)\right)$. Thus, on $h(A), g$ takes each value at most twice (and if twice then at adjacent arguments). As 
$h(A) \in h(U)$ and $h(U)$ is an ultrafilter, there exists $B \in h(U)$ such that $g$ is one-to-one on $B$. As $g$ was an arbitrary finite-to-one function, $h(U)$ is a $Q$-point. But $h$ is finite-to-one, so $h(U)$ is in the cofinal-equivalence class of $U$, which was assumed to contain no $Q$-point.

Since NCF implies that there are no $Q$-points [6, Corollary 15(d)], the proposition just proved clearly yields (a) $\Rightarrow$ (b) in Theorem 5.3 , thus completing the proof of that theorem.

We turn now to the complementary case, where the cofinal-equivalence class under consideration contains a $Q$-point, and we again describe completely the chain of associated ideals.

Proposition 5.7. Let $U$ be a $Q$-point. The $\leqslant$-ordering of the ideals $I(V)$, for $V$ cofinally equivalent to $U$, is canonically isomorphic to the Dedekind completion of the top sky $S$ of the ultrafilter $U$-prod $\omega$. The ideals $I(V)$, for $V$ isomorphic to $U$, are pairwise inequivalent and correspond, under this isomorphism, to the members of $S$.

Proof. Consider first the ultrafilters $V$ isomorphic to $U$, say $V=f(U)$. Then in the ultrapower $U$-prod $\omega$, the elements $u=$ [identity] and $v=[f]$ have types $U$ and $V$ respectively and are in the top sky. We shall show that if $u<v$, then $I(U)<I(V)$. It then follows, by interchanging the roles of $U$ and $V$, that $v<u$ implies $I(V)<I(U)$. Thus, we shall have an order-isomorphism between $\{I(V) \mid V$ isomorphic to $U\}$ and the subset $\{[f] \mid f$ one-to-one $\}$ of $U$-prod $\omega$. As $U$ is a $Q$-point, this latter set is just the top sky $S$, so the ideals $I(V)$ for $V$ isomorphic to $U$ will be ordered as the proposition asserts.

Suppose, therefore, that $u<v$. Then $I(U) \leqslant I(V)$ by Theorem 5.4, and to show that $I(U)<I(V)$ it suffices, by that same theorem, to prove that no model $M^{\prime}$ of arithmetic can contain, in its top sky $S^{\prime}$, two elements $v^{\prime} \leqslant u^{\prime}$ of types $V$ and $U$ respectively. If $M^{\prime}$ were such a model, we could amalgamate it with $U$-prod $\omega$, identifying $v$ with $v^{\prime}$. In the resulting model, $u$ and $u^{\prime}$ are in the same sky, and they are distinct because $u<v=v^{\prime} \leqslant u^{\prime}$. Since they both have type $U$, a $Q$-point, this contradicts Lemma 2.7 so $M^{\prime}$ cannot exist. This completes the proof that the ordering of the $I(V)$ 's for $V$ isomorphic to $U$ is canonically isomorphic to $S$; the isomorphism associates to each element $v$ of $S$ the ideal $I(V)$ where $V$ is the type of $v$.

For $W$ cofinally equivalent but not isomorphic to $U$, the ideal $I(W)$ must, by Corollary 5.5 , be either below all the $I(V)$ 's considered above (i.e. for $V \cong U$ ) or above all these $I(V)$ 's, or equivalent to an $I(V)$, or between two consecutive $I(V)$ 's, or in a gap of the ordering of $I(V)$ 's. (By a gap, we mean that there is no largest $I(V)$ below, and no smallest $I(V)$ above $I(W)$. Note that each element of $S$ has an immediate predecessor and an immediate successor, so the five possibilities listed are exhaustive.) We shall complete the proof of the proposition by showing that only the last of the five possibilities occurs, that every gap in the ordering of the $I(V)$ 's is filled by an $I(W)$, and that any two $I(W)$ 's lying in the same gap are equivalent.

Consider an arbitrary $W$ that is cofinally equivalent but not isomorphic to $U$. Thus, the ultrapower $M=U$-prod $\omega$ does not have an element of type $W$ in its top sky $S$, but some cofinal extension $M^{\prime}$ of it does have such an element $w$ in its top 
sky $S^{\prime} \supset S$. If $v \in S$ and $v<w$, then $v+1<w$ because $w \notin M$. Thus, writing $V$ and $V^{\prime}$ for the types of $v$ and $v+1$, we have $I(V)<I\left(V^{\prime}\right) \leqslant I(W)$, where the strict inequality is from the first part of this proof (as $V$ and $V^{\prime}$ are isomorphic to $U$ ) and the other inequality is from Theorem 5.4. Similarly, if $v \in S$ and $v>w$ then $I(V)>I(W)$. Thus, $I(W)$ fills the gap in the ordering of $I(V)$ 's that corresponds to the gap in $S$ filled by $w$.

Every gap in the ordering of $I(V)$ 's is filled by some $I(W)$, because, by an easy compactness argument, every gap in $S$ is filled in some model of arithmetic extending $M$.

Finally, to show that all $I(W)$ 's that fill the same gap are equivalent, suppose $W$ and $Z$ are two ultrafilters cofinally equivalent to $U$, such that $I(W)<I(Z)$. We shall show that they fill different gaps by finding a $V$ isomorphic to $U$ such that $I(W) \leqslant I(V) \leqslant I(Z)$. As $W$ is cofinally equivalent to $U$ and $U$ is a $Q$-point, Lemma 2.4 shows that $W$ maps to $U$ by a finite-to-one function $f$. Similarly, $Z$ maps to $U$ by a finite-to-one function, and we may assume that it is the same function $f$. (If we had two different functions, we could use an $f$ that agrees with the one on a set in $W$ and agrees with the other on a disjoint set in $Z$. Such sets exist as $W$ and $Z$ are distinct ultrafilters.) Thus, $f(W)=f(Z)=U$. This means that in both $W$-prod $\omega$ and $Z$-prod $\omega$, the equivalence class of $f$ has type $U$, while of course the equivalence class of the identity has type $W$ in the one model and $Z$ in the other. Amalgamate these two models to obtain a model $M^{\prime}$ containing elements $w, z$, $u$, of types $W, Z$, $U$ respectively, and satisfying $f(w)=f(z)=u$.

There cannot be a model of arithmetic containing elements $w^{\prime}, z^{\prime}$, of types $W, Z$ and satisfying both $f\left(w^{\prime}\right)=f\left(z^{\prime}\right)$ and $z^{\prime} \leqslant w^{\prime}$, for in such a model $w^{\prime}$ and $z^{\prime}$ would be in the same sky (with $f\left(w^{\prime}\right)=f\left(z^{\prime}\right)$, as $f$ is finite-to-one) and Theorem 5.4 would then give $I(Z) \leqslant I(W)$ contrary to hypothesis. So the 2-type saying " $x$ has type $W$, $y$ has type $Z, f(x)=f(y)$, and $y \leqslant x$ " is inconsistent with arithmetic. Thus, there are sets $A \in W$ and $B \in Z$ such that, in the standard model of arithmetic,

$$
\forall a \in A \forall b \in B(f(a)=f(b) \Rightarrow a<b) .
$$

Since $f(W)=f(Z)$, we can arrange, by intersecting both $A$ and $B$ with $f^{-1}(f(A)$ $\cap f(B))$ which is in both $W$ and $Z$, that $f(A)=f(B)$. Define $g: f(A) \rightarrow \omega$ by

$$
g(n)=\text { the largest } a \in A \text { with } f(a)=n ;
$$

this makes sense because $f$ is finite-to-one. Then obviously

$$
(\forall a \in A) a \leqslant g(f(a)) .
$$

Furthermore, given any $n \in f(A)=f(B)$, we can apply (1) to $a=g(n)$, obtaining, as $f(a)=n$,

$$
(\forall b \in B)(n=f(b) \Rightarrow g(n)<b)
$$

which simplifies to

$$
(\forall b \in B) g(f(b))<b .
$$

Both (2) and (3), being true in the standard model of arithmetic, are true in all models of arithmetic, in particular in our model $M^{\prime}$ containing $w, z$, and $u$ as above. The type $W$ of $w$ contains $A$, so (2) is applicable with $a=w$. The type $Z$ of $z$ 
contains $B$, so (3) is applicable with $b=z$. Since $f(w)=f(z)=u$, we obtain

$$
w \leqslant g(f(w))=g(u)=g(f(z))<z .
$$

Since $g$ is one-to-one on the set $f(A) \in U$, the type $V=g(U)$ of $g(u)$ is isomorphic to $U$. And the inequalities $w \leqslant g(u) \leqslant z$ imply, by Theorem 5.4, that $I(W) \leqslant I(V)$ $\leqslant I(Z)$, as required.

6. Slenderness classes of groups. We shall apply the results of the previous section, whose notation we continue to use, to show the relevance of NCF to the ordering $\mathbf{P}$ studied by Göbel and Wald [12, 13]. Although we shall not need the connection between this ordering and slenderness of groups, we include a brief summary of it for the sake of completeness and motivation. For details, see [12, 13] and the papers cited there.

A growth type is defined $[12,23]$ to be a subset of $\omega \nearrow \omega$, closed under pointwise addition, closed downward, and containing all bounded elements of $\omega \nearrow \omega$. (The last requirement can be replaced by "nonempty" if one considers only positive-valued functions; we consider all of $\omega \nearrow \omega$ for the sake of coherence with the previous section.) In the presence of downward closure, closure under pointwise addition is clearly equivalent to closure under pointwise maximum and doubling. Thus, a growth type is an ideal in $\omega \nearrow \omega$ that is closed under doubling. Of the ideals discussed in the last section, $B$ and $\omega \nearrow \omega$ are growth types. $L$ is not a growth type but is equivalent (in the sense of the previous section) to the growth type

$$
+L=\{f \in \omega \nearrow \omega \mid f \text { is majorized by a linear function }\},
$$

because $L \subseteq+L$ and $+L$ is dominated by the function $n \mapsto n^{2}$.

Quite generally, any ideal $I$ generates a growth type,

$$
+I=\{f \in \omega \nearrow \omega \mid \text { for some } c \in \omega \text { and some } g \in I, c \cdot g \text { majorizes } f\},
$$

which is the smallest growth type that includes $I$. In particular, every nonprincipal ultrafilter $U$ yields a growth type

$$
+I(U)=\{f \in \omega \nearrow \omega \mid f \text { is majorized by a linear function on some set in } U\} .
$$

Since growth types are ideals, the collection of growth types is preordered by the relation $\leqslant$ studied in the last section. The partially ordered set of equivalence classes of growth types is called P. It was introduced in [12] and studied further in [13] because of the following connection with group theory.

Each growth type $T$ determines a subgroup [ $T$ ] of the product $\mathbf{Z}^{\omega}$ of countably many copies of the additive group $\mathbf{Z}$ of integers, namely,

$$
[T]=\{f: \omega \rightarrow \mathbf{Z} \mid \text { the function } n \mapsto \max \{|f(i)| \mid i \leqslant n\} \text { is in } T\} .
$$

Subgroups that arise in this way are called monotone subgroups of $\mathbf{Z}^{\omega}$. A group $G$ is said to be $[T]$-slender if every homomorphism $[T] \rightarrow G$ annihilates all but finitely many of the functions $f_{n}$ defined by the Kronecker delta, $f_{n}(i)=\delta_{n, i}$. (This definition was motivated by Specker's theorem [23] that $\mathbf{Z}$ is $\mathbf{Z}^{\omega}$-slender.) It is proved in [12] that $T_{1} \leqslant T_{2}$ if and only if every [ $\left.T_{1}\right]$-slender group is also [ $\left.T_{2}\right]$-slender. Thus, the study of $\mathbf{P}$ amounts to the study of the inclusion ordering on the slenderness classes of groups obtained from various growth types. 
The top and bottom of the ordering $\mathbf{P}$ of growth types have even simpler descriptions than for ideals. Using the corresponding results for ideals, we find that $B$ is the smallest growth type (since $B_{k}$ is not a growth type), that $B$ is not equivalent to any other growth type, that $+L$ is an immediate successor of $B$, that the growth types equivalent to $+L$ are the dominated ones other than $B$, and that all undominated growth types are above $L$. The growth type $\omega \nearrow \omega$ is not equivalent to any other (since growth types, unlike ideals, are always closed under finite alterations of functions) and is, of course, at the top of the ordering. The growth types $+I(U)$, which are clearly undominated but $<\omega \nearrow \omega$, for all ultrafilters $U$, are cofinal in $\mathbf{P}-\{\omega \nearrow \omega\}$; indeed, they are cofinal among the ideals $\langle\omega \nearrow \boldsymbol{\omega}$ because the $I(U)$ 's are (Proposition 5.2) and $I(U) \subseteq+I(U)$. The growth types $I(U)$ were introduced in [13] to show that $\mathbf{P}$ has at least 4 members.

LEMMA 6.1. For any ideals, if $I \leqslant J$ then $+I \leqslant+J$.

Proof. If $r$ is as in the definition of $I \leqslant J$, then the same $r$ witnesses $+I \leqslant+J$.

Proposition 6.2. For any ultrafilters $U$ and $V$, the following are equivalent.

(a) $+I(U)$ and $+I(V)$ are comparable in $\mathbf{P}$.

(b) $+I(U)$ and $+I(V)$ have an upper bound in $\mathbf{P}-\{\omega \nearrow \omega\}$.

(c) $U$ and $V$ are cofinally equivalent.

Proof. (a) $\Rightarrow$ (b) is trivial

(b) $\Rightarrow$ (c). The upper bound, considered as an ideal, is $\leqslant I(W)$ for some nonprincipal ultrafilter $W$. Then both $I(U)$ and $I(V)$ are comparable with (in fact $\leqslant$ ) $I(W)$, so both $U$ and $V$ are cofinally equivalent with $W$, hence with each other.

$($ c) $\Rightarrow($ a). $I(U)$ and $I(V)$ are comparable, by Corollary 5.5, and Lemma 6.1 gives (a).

THEOREM 6.3. The following are equivalent:

(a) $N C F$.

(b) The growth types $+I(U)$ are all equivalent.

(c) The growth types $+I(U)$ are all comparable in $\mathbf{P}$.

(d) $\mathbf{P}-\{\omega \nearrow \omega\}$ has a top element.

Proof. The preceding proposition gives (a) $\Leftrightarrow$ (c) and (d) $\Rightarrow$ (a). Obviously, (b) $\Rightarrow$ (c). To complete the proof, it suffices to show that (a) implies both (b) and (d). Assume (a). By Theorem 5.3 and Proposition 5.2, all of the ideals $I(U)$ are equivalent, and their common equivalence class is at the top of the ideal ordering minus the class of $\omega \nearrow \omega$. Since $+I(U)$ includes $I(U)$ and is not equivalent to $\omega \nearrow \omega$, all of the $+I(U)$ 's must be in this equivalence class also. Now (b) and (d) are obvious.

The remainder of this section is devoted to a complete description of the ordering of the growth types $+I(U)$ for ultrafilters $U$. By Proposition 6.2, this ordering is a disjoint union of chains, where the chains correspond to the cofinal-equivalence 
classes of ultrafilters, and where growth types from different chains are incomparable and in fact have no common upper bound. It thus suffices to consider each chain, and its associated cofinal-equivalence class, separately.

Proposition 6.4. If a cofinal-equivalence class contains no Q-point, then the corresponding chain in $\mathbf{P}$ consists of a single element.

Proof. For $U$ and $V$ in such a cofinal-equivalence class, the ideals $I(U)$ and $I(V)$ are equivalent, by Proposition 5.6. Then the growth types $+I(U)$ and $+I(V)$ are equivalent, by Lemma 6.1.

We turn now to the more difficult case of a cofinal-equivalence class that contains a $Q$-point. The ordering of the $I(U)$ 's, for $U$ in such a class, is described by Proposition 5.7 as being canonically isomorphic to the Dedekind completion of the top sky of the $Q$-point ultrapower. Lemma 6.1 implies that the ordering of the $+I(U)$ 's is obtained from that of the $I(U)$ 's by collapsing certain intervals to points. That is, $\leqslant$ is preserved by the passage from $I$ to $+I$, but $<$ need not be preserved. Our task is to determine which inequivalent pairs among the $I(U)$ 's become equivalent when we apply + to them.

Fix a $Q$-point $U$ and an element $u$ of type $U$ in some model $M$ of arithmetic; for example we could take $u=[$ id] in $U$-prod $\omega$. Let $V$ be the type of $n \cdot u$ for an arbitrary standard positive integer $n$. Then we can show that $+I(V)$ and $+I(U)$ are equivalent. Of course $+I(U) \leqslant+I(V)$ by Theorem 5.4 and Lemma 6.1 so it suffices to prove the reverse inequality, which reduces to $I(V) \leqslant+I(U)$ by Lemma 6.1 , since $++I=+I$. Thus, what must be shown is

$$
\begin{aligned}
& (\exists r \in \omega \nearrow \omega)(\forall A \in V)(\exists B \in U)(\exists c \in \omega) \\
& \quad(\forall \text { sufficiently large } x \in \omega) \operatorname{next}(A, x) \leqslant c \cdot \operatorname{next}(B, r(x)) .
\end{aligned}
$$

To show this, take $r$ to be the identity and, given $A \in V$ so $n \cdot u \in A$, take $B=\{m \in \omega \mid n \cdot m \in A\}$. Thus $u \in B$ so $B \in U$. Finally take $c=n$. Then for any $x, c \cdot \operatorname{next}(B, r(x))=n \cdot \operatorname{next}(B, x)$ is an element of $n \cdot B \subseteq A$ greater than or equal to $x$ and therefore greater than or equal to $\operatorname{next}(A, x)$, as required.

Under the identification of the chain of $I(W)$ 's, $W$ cofinally equivalent to $U$, with the Dedekind completion of the constellation of $u$, as given by Proposition 5.7, the + operation has just been shown to identify $u$ with all its multiples $n \cdot u$ by standard positive integers $n$. Since + preserves $\leqslant$, it identifies $u$ with all $v$ that lie between $u$ and any of these $n \cdot u$. It also identifies $u$ with all elements that lie between $\lfloor u / n\rfloor$ and $u$ for any $n$, since the type of $\lfloor u / n\rfloor$ is (by the remarks preceding Lemma 2.7) a $Q$-point that could have been used in place of $U$ in the preceding discussion. Thus, the + operation collapses the whole interval, in the Dedekind completion of the constellation of $u$, of elements that differ from $u$ by only a finite factor.

We shall show that the right endpoint of this interval (in the Dedekind completion) is also identified with $u$ but the left endpoint is not. It follows, of course, that nothing below the left endpoint is identified with $u$ (as + preserves order). It also follows that nothing above the right endpoint is identified with $u$. To see this, let $w$ 
be this right endpoint and let $v>w$. There is a $u^{\prime}$ in the constellation of $u$ (not merely in the Dedekind completion) between $w$ and $v$. No $\left\lfloor u^{\prime} / n\right\rfloor$ can be $<w$, for, by definition of $w$, its set of predecessors is closed under multiplication by any standard $n$. So the left endpoint of the interval of elements differing from $u^{\prime}$ by a finite factor is $\geqslant w$. (In fact, it is $>w$, but we do not need this.) Nothing below this endpoint gets identified with $u^{\prime}$; in particular, $u$ is not identified with $u^{\prime}$, nor, a fortiori, with $v$.

Thus, once we verify our claims about identifications involving the endpoints, we shall know exactly which intervals in the chain of ideals (corresponding to our fixed cofinal-equivalence class of ultrafilters) are collapsed to form single elements in the chain of growth types. In terms of the isomorphism with the Dedekind completion of the constellation of $u$, they are exactly the intervals gotten by taking an arbitrary element $v$ of that constellation, forming the smallest interval that contains $\mid v / n\rfloor$ and $n \cdot v$ for all standard positive $n$, and adding to this interval its right endpoint. Clearly each element of the constellation of $u$ is in a unique such interval. Some elements of the Dedekind completion are in no such interval; these are not identified with anything else under the + operation (because every nontrivial interval in the Dedekind completion contains points from the constellation of $u$ ).

It therefore remains only to verify our assertions about identifications at the endpoints of our intervals.

Consider first the right endpoint $I(W)$ of the interval containing $I(U)$, where $U$ is our fixed $Q$-point. $W$ is the type of an element $w$, in some extension $M^{\prime}$ of $M$, such that $w>n \cdot u$ for all finite $n$ but $w<u^{\prime}$ for all $u^{\prime}$ in the constellation of $u$ such that $u^{\prime}>n \cdot u$ for all $n$. That is, $w$ lies in the gap, of the constellation of $u$, that comes immediately after the elements $n \cdot u$. Our objective is to show that $+I(W) \leqslant+I(U)$, which reduces, as before, to $I(W) \leqslant+I(U)$, i.e.,

$$
(\exists r \in \omega \nearrow \omega)(\forall A \in W)(\exists B \in U)(\exists c \in \omega)
$$

$(\forall$ sufficiently large $x \in \omega) \operatorname{next}(A, x) \leqslant c \cdot \operatorname{next}(B, r(x))$.

To establish this, first fix an $X \in U$ such that, whenever $x<y$ are in $X$, then $x^{2}<y$. Such an $x$ exists, by Lemma 2.3 since $U$ is a $Q$-point. Since $U$ is the type of $u$, we have $u \in X$. All elements of $X$ above $u$ are, by our choice of $X$, also above $u^{2}$ which is $>n \cdot u$ for all finite $n$ (as $u$ is infinite) and therefore $>w$ (because $w$ fills the gap right after the $n \cdot u$ 's). Thus, $u$ is the last element of $X$ before $w$.

Now let $r$ be the identity function and let an arbitrary $A \in W$ be given; we seek $B$ and $c$ as in the formula defining $I(W) \leqslant+I(U)$. As $w$ has type $W$, we have $w \in A$ and therefore $\operatorname{next}(A, u) \leqslant w$. But $\operatorname{next}(A, u)$ is in the constellation of $u$ (being defined from $u$ and being $\geqslant u$ ), so $\operatorname{next}(A, u) \leqslant n \cdot u$ for some finite $n$. Set $c=n$ and set

$$
B=\{b \in X \mid \operatorname{next}(A, b) \leqslant c \cdot b\} .
$$

This $B$ is in $U$, because $\operatorname{next}(A, u) \leqslant c \cdot u$ and $U$ is the type of $u$. For an arbitrary $x \in \omega$, we have

$$
\operatorname{next}(A, x) \leqslant \operatorname{next}(A, \operatorname{next}(B, x)) \leqslant c \cdot \operatorname{next}(B, x),
$$

as required. This completes the proof that $+I(W) \leqslant+I(U)$. 
We turn now to the left endpoint $I(V)$ of the interval containing $I(U) . V$ is the type of an element $v$, in some extension of $M$, such that $v$ fills the gap, of the constellation of $u$, immediately before the elements $\lfloor u / n\rfloor$ for finite $n$. Thus, an element of the constellation of $u$ is $>v$ if and only if it is $\geqslant\lfloor u / n\rfloor$ for some finite $n$. Our objective is to prove $+I(U) \nless+I(V)$, i.e.,

$$
(\forall r \in \omega \nearrow \omega)(\exists A \in U)(\forall B \in V)(\forall c \in \omega)
$$

( $\exists$ arbitrarily large $x \in \omega) \operatorname{next}(A, x)>c \cdot \operatorname{next}(B, r(x))$.

As in the previous argument, fix $X \in U$ such that whenever $x<y$ are in $X$ then $x^{2}<y$. Then $u \in X$ and the next smaller element of $X$ is $\leqslant\lfloor\sqrt{u}\rfloor$ which is $<\lfloor u / n\rfloor$ for all finite $n$ (as $u$ is infinite) and in the constellation of $u$, hence $<v$. So $u=\operatorname{next}(X, v)$.

For any given $r \in \omega \nearrow \omega$, let $A \in U$ be a subset of $X$ such that

$$
p<q \text { in } A \Rightarrow \operatorname{next}(X, r(p+1))<q \text {; }
$$

such an $A$ exists by Lemma 2.3 because $U$ is a $Q$-point. We shall show that this $A$ has the properties required in the formula expressing $+I(U) \nless+I(V)$. Let arbitrary $B \in V$ and $c \in \omega$ be given. We must find arbitrarily large values of $x$ for which next $(A, x)>c \cdot \operatorname{next}(B, r(x))$.

Since $c$ is finite, $v<\lfloor u / c\rfloor$, so

$$
c v<u=\operatorname{next}(X, v) \in A \text {, }
$$

where we have also used that $A$ is in the type $U$ of $u$. Thus, the type $V$ of $v$ contains the set

$$
B^{\prime}=\{b \in B \mid c \cdot b<\operatorname{next}(X, b) \in A\},
$$

since it contains $B$.

Consider an arbitrary $b \in B^{\prime}$. Let $q=\operatorname{next}(X, b)$. As $b \in B^{\prime}$, we have $q \in A$, and, since $A \subseteq X$, we infer that $q=\operatorname{next}(A, b)$. Let $p$ be the largest element of $A$ that is $<q$, and let $x=p+1$. Our choice of $A$ ensures that $\operatorname{next}(X, r(x))<q$. This inequality together with $q=\operatorname{next}(X, b)$ implies $r(x)<b$. Thus, $\operatorname{next}(B, r(x))$ $\leqslant b$, and therefore

$$
c \cdot \operatorname{next}(B, r(x)) \leqslant c \cdot b<\operatorname{next}(X, b)=q=\operatorname{next}(A, x),
$$

where the strict inequality comes from the fact that $b \in B^{\prime}$ and the last equality comes from the choice of $p$ and $x$.

Since $B^{\prime}$ is in $V$, it has arbitrarily large members $b$, and these clearly yield arbitrarily large values of $x$. Since all such values of $x$ have been shown to satisfy $\operatorname{next}(A, x)>c \cdot \operatorname{next}(B, r(x))$, the proof of $+I(U) \nless+I(V)$ is complete.

This completes the computation of the order type of the chain of growth types associated to a cofinal-equivalence class that contains a $Q$-point. The description can be slightly simplified by the following considerations. Each of the intervals $\bigcup_{n \in \omega}[\lfloor u / n\rfloor, n \cdot u]$, which, together with its right endpoint, was collapsed by the + operation, contains elements of $M$ of the form $2^{v}$, with $v$ in the constellation of $u$; indeed, we need only take $v=\lceil\log u\rceil$, where " $\log$ " refers to base 2 , and we have $u \leqslant 2^{v}<2 u$. Under the logarithm operation, the interval under discussion corresponds to the interval $\bigcup_{n \in \omega}[v-n, v+n]$, i.e., to the galaxy of $v$. Thus, the results of the preceding computation can be put into the following form. 
Proposition 6.5. Let $U$ be a Q-point. The chain of growth types associated to the cofinal-equivalence class of $U$ is order-isomorphic to the chain obtained from the Dedekind-completion of the top sky of $U$-prod $\omega$ by collapsing each galaxy together with its right endpoint.

Corollary 6.6. (a) $\mathbf{P}-\{\omega \nearrow \omega\}$ has a maximal element if and only if there is a nonprincipal ultrafilter that cannot be mapped to any $Q$-point by any finite-to-one function.

(b) Every element of $\mathbf{P}-\{\omega \nearrow \omega\}$ is below a maximal one if and only if there are no Q-points.

Proof. Both assertions follow from Propositions 6.4 and 6.5 and the fact that the $+I(U)$ 's are cofinal in $\mathbf{P}-\{\omega \nearrow \omega\}$. For (a), recall (Lemma 2.4) that an ultrafilter is cofinally equivalent to a $Q$-point if and only if it is mapped to that $Q$-point by a finite-to-one function.

Let $S$ be the set of countable transfinite sequences of zeros and ones,

$$
S=\{f \mid \text { for some countable ordinal } \alpha, f: \alpha \rightarrow\{0,1\}\},
$$

linearly ordered lexicographically. Using the saturation property [1] of ultrapowers, it is easy to embed $S$ order-isomorphically into the top sky of $U$-prod $\omega$ for any ultrafilter $U$; in addition, one can arrange that distinct elements of $S$ are mapped into distinct galaxies of $U$-prod $\omega$. It follows that the chain described in Proposition 6.5 includes a copy of the Dedekind-completion of $S$, which is easily seen to have cardinality at least $2^{\aleph_{1}}$. Thus, if there is a $Q$-point, then $\mathbf{P}$ has cardinality at least $2^{\aleph_{1}}$. Göbel and Wald [13] obtained the stronger conclusion that $\mathbf{P}$ has cardinality $2^{2^{\mathrm{N}_{0}}}$ from the stronger assumption that Martin's axiom holds.

\section{REFERENCES}

1. J. Bell and A. B. Slomson, Models and ultraproducts, North-Holland, 1969.

2. D. Bellamy, A non-metric indecomposable continuum, Duke Math. J. 38 (1971), 15-20.

3. __ Indecomposable continua with one and two composants, Fund. Math. 101 (1978), 129-134.

4. A. Blass, The intersection of non-standard models of arithmetic, J. Symbolic Logic 37 (1972), 103-106.

5. A. Blass and G. Weiss, A characterization and sum decomposition of operator ideals, Trans. Amer. Math. Soc. 246 (1978), 407-417.

6. A. Blass, Near coherence of filters, I: Cofinal equivalence of models of arithmetic, Notre Dame J. Formal Logic 27 (1986), 579-591.

7. A. Blass and S. Shelah, There may be simple $P_{\aleph_{1}}$ and $P_{\aleph_{2}}$ points and the Rudin-Keisler ordering may be downward directed, Ann. Pure Appl. Logic (to appear).

8. A. Blass, A model-theoretic view of some special ultrafilters, Logic Colloquium '77 (A. Macintyre, L. Pacholski, and J. Paris, eds.), North-Holland, 1978, pp. 79-90.

9. A. Brown, C. Pearcy, and N. Salinas, Ideals of compact operators on Hilbert space, Michigan Math. J. 19 (1971), 373-384.

10. W. W. Comfort and S. Negrepontis, Theory of ultrafilters, Springer-Verlag, 1974.

11. L. Gillman and M. Jerison, Rings of continuous functions, Van Nostrand, 1960.

12. R. Göbel and B. Wald, Wachstumstypen und schlanke Gruppen, Symposia Math. 23 (1979), 201-239.

13. __ Martin's axiom implies the existence of certain slender groups, Math. Z. 172 (1980), $107-121$.

14. A. S. Kechris, On a notion of smallness for subsets of the Baire space, Trans. Amer. Math. Soc. 229 (1977), 191-207.

15. J. Mioduszewski, On composants of $\beta R-R$, Proc. Conf. Topology and Measure. I (Zinnowitz, 1974) (J. Flachsmeyer, Z. Frolik and F. Terpe, eds.), Ernst-Moritz-Arndt-Universität zu Greifwald, 1978, pp. 257-283. 
16. An approach to $\beta \mathbf{R} \backslash \mathbf{R}$ Topology, Colloq. Math. Soc. János Bolyai 23 (Á. Császár, ed.), North-Holland, 1980, pp. 853-854.

17. C. Puritz, Ultrafilters and standard functions in non-standard arithmetic, Proc. London Math. Soc. (3) 22 (1971), 705-733.

18. _ Skies, constellations, and monads, Contributions to Non-Standard Analysis (W. A. J. Luxemburg and A. Robinson, eds.), North-Holland, 1972, pp. 215-243.

19. M. E. Rudin, Composants and $\beta N$, Proc. Washington State Univ. Conf. General Topology 1970, pp. $117-119$

20. J. Saint-Raymond, Approximations des sous-ensembles analytiques par l'intérieur, C. R. Acad. Sci. Paris 281 (1975), A85-A87.

21. R. Schatten, Norm ideals of completely continuous operators, Springer-Verlag, 1960.

22. R. C. Solomon, Families of sets and functions, Czechoslovak Math. J. 27 (1977), 556-559.

23. E. Specker, Additive Gruppen von Folgen ganzer Zahlen, Portugal. Math. 9 (1950), 131-140.

Department of Mathematics, Pennsylvania State University, University Park, Pennsylvania 16802

Current address: Department of Mathematics, University of Michigan, Ann Arbor, Michigan 48109 